\title{
TRANSIENT VELOCITY AND STEADY STATE ENTROPY GENERATION IN A MICROFLUIDIC COUETTE FLOW CONTAINING CHARGED NANO PARTICLES
}

\author{
R.S.R. GORLA* \\ Department of Mechanical Engineering, Cleveland State University \\ Cleveland, Ohio-44115, USA \\ E-mail:r.gorla@csuohio.edu \\ B.J. GIREESHA \\ Department of Mechanical Engineering, Cleveland State University \\ Cleveland, Ohio-44115, USA \\ Department of Studies and Research in Mathematics, Kuvempu University \\ Shankarghatta 577451, Shimoga, Karnataka, INDIA \\ E-mail: g.bijjanaljayanna@csuohio.edu
}

\begin{abstract}
An analysis has been provided to determine the transient velocity and steady state entropy generation in a microfluidic Couette flow influenced by electro-kinetic effect of charged nanoparticles. The equation for calculating the Couette flow velocity profile is derived for transient flow. The solutions for momentum and energy equations are used to get the exact solution for the dimensionless velocity ratio and dimensionless entropy generation number. The effects of the dimensionless entropy generation number, Bejan number, irreversibility ratio, entropy generation due to fluid friction and due to heat transfer on dimensionless time, relative channel height, Brinkman number, dimensionless temperature ratio, nanoparticle volume fraction are analyzed.
\end{abstract}

Key words: transient velocity, steady state entropy generation, microfluidic Couette flow, charged nanoparticles, Bejan number and Brinkman number.

\section{Introduction}

In recent years, microfluidics or micro-fluid mechanics have become an attractive area of research. The importance of microfluidics arises from the new applications of micro-scale electromechanical systems (MEMS) that encounter mass and heat transfer, such as micro-pumps, micro-turbines and microrefrigerators. Extensive experimental and theoretical studies were made in this area in order to understand the fluid behavior within this micro devices. Reynolds numbers in microfluidic systems are usually small, i.e., usually below 0.1 . Microfluidics deals with the science and technology of fluid flows over micron or sub-micron length scales. In addition, due to the small scales of the channels, the surface-to-volume ratio is high causing surface effects such as wettability or surface charges to be more important than in macroscopic systems.

Nanofluids, a new class of nano-engineered liquid solutions of colloidal particles with a diameter of $1-100 \mathrm{~nm}$, have shown a great energy savings potential and attractive properties for applications such as energy, bio and pharmaceutical industry, and chemical, electronic, environmental, material, medical and thermal engineering, among others. Based on these applications (Choi et al., 2011) theoretically studied the behavior of a microfluidic Couette flow of nanofluids composed of negatively-charged nanoparticles

\footnotetext{
" To whom correspondence should be addressed
} 
dispersed in aqueous $\mathrm{NaCl}$ solutions. They derived the equation for calculating the Couette flow velocity profiles and also calculated the induced electric field and velocity profiles as a function of key parameters including nanoparticle size and volume fraction. They showed that nanofluids consisting of negatively charged nanoparticles suspended in aqueous $\mathrm{NaCl}$ solutions show significantly different velocity profiles compared to aqueous $\mathrm{NaCl}$ solutions containing no nanoparticles. Viscoelastic flow around a confined cylinder at high Deborah numbers is studied using microfluidic channels by Kenney et al. (2013). This study was concentrated on new flow instabilities for large Deborah number flows that occur both downstream and upstream of the cylinder and also on the role of inertia and elasticity in the flow. Omowunmi and Yuan (2013) performed a numerical study on the role of elongational viscosity in time-dependent non-linear dynamics of polymer solutions in micro-fluidic contraction flow. By extending the work of Groisman and Steinberg (1998) to microfluidic channels, Pathak et al. (2004) conducted flow visualization to study the mechanism underlying a purely elastic flow instability for a Poiseuille flow in a micro channel having a zigzag path (curved streamlines) and quantitatively investigated its implications for fluid mixing (studied by fluorescence microscopy) in a micro channel. Soong and Wang (2003) investigated the electro-kinetic effects on liquid flow and heat transfer in a flat micro channel of two parallel plates under asymmetric boundary conditions including wall-sliding motion, unequal zeta potentials, and unequal heat flux on two walls. Zahid et al. (2007) studied a Couette-Poiseuille flow of a gas in long micro channels.

In order to improve the performance of micro-fluid mechanics in energy utilization, recently the entropy generation analysis methodology which is based on the second-law of thermodynamics, has been effectively used. The open literature shows a very small number of papers that deal with entropy generation related problems in the field of microfluidics. Ejtehadi et al. (2012) numerically analyzed compressibility and rarefaction effects on entropy generation in a micro/nano Couette flow using the direct simulation Monte Carlo (DSMC) method. Under more general conditions Ibanez and Cuevas (2010) analyzed, the thermal behavior of viscous fluid flow in a parallel wall microchannel subjected to electromagnetic interaction. Further, they also explored the influence of these conditions on the entropy generation rate in the microdevice and determined conditions under which this quantity is minimized. Haddad et al. (2004) numerically investigated the entropy generation due to steady laminar forced convection fluid flow through parallel plate's microchannel and discussed the effect of Knudsen, Reynolds, Prandtl, Eckert numbers and the nondimensional temperature difference on entropy generation within the microchannel. With the aid of the lattice Boltzmann method, Chen and Tian (2010) studied the characteristics of entropy generation due to heat transfer and friction in transient state as well as in steady state for thermal micro-Couette flows in slip regime. Chen (2005) carried out an analytical investigation on entropy generation and transfer in microchannel flow by considering different boundary conditions and for an arbitrary channel shape. Recently, Abbassi (2007) used an analytical approach to solve forced convection problem across heated microchannel heat sink (MCHS), in a porous medium model based on extended Darcy equation for fluid flow and two-equation model for heat transfer. They proved that there is an optimized value for porosity at which the entropy generation rate reaches its minimum magnitude.

Based on the above studies the objective of the present study is to discuss the transient velocity and steady state entropy generation in a microfluidic Couette flow containing charged nanoparticles. The effects of the dimensionless entropy generation number, Bejan number, irreversibility ratio, entropy generation due to fluid friction, entropy generation due to heat transfer on dimensionless time, relative channel height, Brinkman number, dimensionless temperature ratio, nanoparticle volume fraction are studied.

\section{Mathematical formulation and solution analysis}

Consider a steady, laminar flow of an incompressible fluid between two parallel plates of a microchannel of width $2 h$ as shown in Fig. 1a. The Couette flow is due to the movement of the upper plate with uniform velocity $(U)$ along the $x$-axis, while the lower plate is stationary. Figure 1 shows the EDL at the solid-liquid interface. 


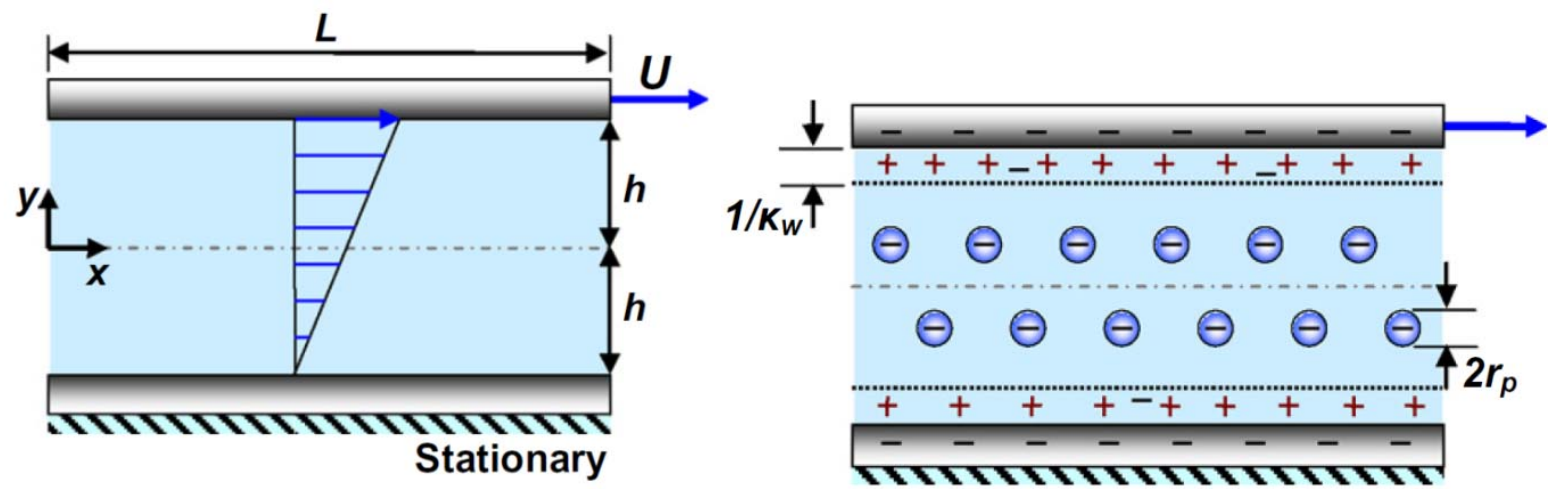

Fig.1. Schematic diagram of the flow.

We have made the following assumptions to study the Couette flow velocity profile.

1. The flow is one dimensional i.e., $u=u(y)$.

2. Nanoparticles are mono disperse, rigid spheres with radius $r_{p}$ it ranges between 3-50 $\mathrm{nm}$, and homogeneously distributed in the solution.

3. Interaction between charged nanoparticles in the suspension is negligible because the concentration of nanoparticles is less than 0.4 vol. $\%$.

The equation governing one-dimensional flow in the presence of body force induced by the flow of charged nanoparticles in a nanofluid is given as follows

$$
\rho \frac{\partial u}{\partial t}=\frac{\partial^{2} u}{\partial y^{2}}-\left(\varepsilon k^{2} \zeta_{w} E_{x}\right)\left[\frac{\cosh k y}{\cosh k h}\right] .
$$

Initial and boundary conditions for the present formulation are

$$
\begin{aligned}
& u(0, y)=0, \\
& u(t, h)=U, \quad u(t,-h)=0 .
\end{aligned}
$$

To find the solution of the governing problem here we have implemented the variable separable method. So let

$$
u(t, y)=u_{s}(y)+u_{t}(t, y) .
$$

By substituting Eq.(2.3) in Eqs (2.1) and (2.2) one can get the equation for the velocity as

$$
\rho \frac{\partial u_{t}}{\partial t}=\frac{\partial^{2} u_{t}}{\partial y^{2}}+\frac{\partial^{2} u_{s}}{\partial y^{2}}-\left(\varepsilon k^{2} \zeta_{w} E_{x}\right)\left[\frac{\cosh \left(k_{w} y\right)}{\cosh \left(k_{w} h\right)}\right] .
$$

The boundary conditions are

$$
u(-h)=0, \quad u(h)=U .
$$

By solving Eq.(2.4) along with the boundary conditions (2.5) we get the solution for steady state velocity $u_{s}$ as 


$$
\frac{u_{s}}{U}=\frac{1}{2}\left(\frac{y}{h}+1\right)-\left(\frac{\Omega_{E} E_{x}}{U}\right)\left[1-\frac{\cosh \left(k_{w} y\right)}{\cosh \left(k_{w} h\right)}\right]
$$

where $\quad \Omega_{E}=\frac{\varepsilon \zeta_{w}}{\mu}, E_{x}=\frac{U f_{v}}{r_{p}^{2} \sigma_{e f f} h} \cdot h=20 \mu m, \quad r_{p}=5 \mathrm{~nm}, \quad \varepsilon=6.95 * \frac{10^{-10} \mathrm{C}^{2}}{\mathrm{Nm}^{2}}, \quad \lambda_{w}=1000 \mathrm{~nm}$, $\mu_{f}=8.9 * \frac{10^{-4} \mathrm{Ns}}{m^{2}}, \zeta_{w}=-62.2 \mathrm{mV}, k_{w}=\frac{1}{\lambda_{w}}=10^{6} / \mathrm{m}, \sigma_{\text {eff }}=6.42 * 10^{-3} \mathrm{~S} / \mathrm{m}$.

Again using Eq.(2.3) in Eq.(2.1) we get the equation for transient velocity $u_{t}(t, y)$ as;

$$
\rho \frac{\partial u_{t}}{\partial t}=\frac{\partial^{2} u_{t}}{\partial y^{2}}
$$

Corresponding initial and boundary conditions will become

$$
\begin{aligned}
& u_{t}(0, y)=-u_{s}(y), \\
& u_{t}(t,-h)=0, \quad u_{t}(t, h)=0 .
\end{aligned}
$$

The transformation $Y=y+h$ will reduce Eq.(2.7) and initial and boundary conditions (2.8) into the following form

$$
\rho \frac{\partial u_{t}}{\partial t}=\frac{\partial^{2} u_{t}}{\partial Y^{2}} .
$$

Initial and boundary conditions defined as in Eq.(2.8) will become

$$
\begin{array}{ll}
u_{t}(0, Y)=-u_{s}(Y) & \text { (Initial condition) } \\
u_{t}(t, 0)=0, & u_{t}(t, 2 h)=0 \quad \text { (boundary conditions) }
\end{array}
$$

Using separation of variables method and orthogonal property, the solution of Eq.(2.9) along with initial and boundary conditions as in Eqs (2.10) and (2.11) we get,

$$
u_{t}=\sum_{n=1}^{\infty} \frac{2 U \cos n \pi}{n \pi}\left[\begin{array}{c}
+\left(2 \Omega_{E} E_{x}\right)(1-\cos n \pi) \\
\left(\frac{1}{n \pi}-\frac{n \pi}{n^{2} \pi^{2}+4 h^{2} k_{w}^{2}}\right)
\end{array}\right]\left(e^{-\left(\frac{n^{2} \pi^{2}}{4 h^{2}} * v t\right)}\right)\left(\sin \left[\frac{n \pi}{2}\left(\frac{y}{h}+1\right)\right]\right) .
$$

Let $\frac{v t}{h^{2}}=\tau$, as a dimensionless time in $e^{-\left(\frac{n^{2} \pi^{2}}{4 h^{2}} * v t\right)}, h=20 \mu \mathrm{m}, k_{w}=\frac{10^{6}}{m}$, for $10^{-7} \mathrm{M}$ solution. Now the solution for the transient velocity will become 


$$
\frac{u_{t}}{U}=\sum_{n=1}^{\infty}\left[\begin{array}{c}
\frac{2 \cos n \pi}{n \pi}+\left(\frac{2 \Omega_{E} E_{x}}{U}\right)(1-\cos n \pi) \\
\left(\frac{1}{n \pi}-\frac{n \pi}{n^{2} \pi^{2}+4 h^{2} k_{w}^{2}}\right)
\end{array}\right]\left(e^{-\left(\frac{n^{2} \pi^{2}}{4} *^{2}\right)}\right)\left(\sin \left[\frac{n \pi}{2}\left(\frac{y}{h}+1\right)\right]\right) .
$$

For the sake of convenience and for rapid convergence of the series we have considered the following first six terms of the series,

$$
\begin{aligned}
& \frac{u_{t, 1}}{U}=\left[(-0.6356)+\left(\frac{4 \Omega_{E} E_{x}}{U}\right)(0.3163)\right]\left(e^{-\left(2.467^{*} \tau\right)}\right)\left(\sin \left[\frac{\pi}{2}\left(\frac{y}{h}+1\right)\right]\right), \\
& \frac{u_{t, 2}}{U}=[(0.3183)]\left(e^{-\left(9.869^{*} \tau\right)}\right)\left(\sin \left[\pi\left(\frac{y}{h}+1\right)\right]\right), \\
& \frac{u_{t, 3}}{U}=\left[(-0.2122)+\left(\frac{4 \Omega_{E} E_{x}}{U}\right)(0.1005)\right]\left(e^{-\left(22.2^{*} \tau\right)}\right)\left(\sin \left[\frac{3 \pi}{2}\left(\frac{y}{h}+1\right)\right]\right), \\
& \frac{u_{t, 4}}{U}=[(0.1591)]\left(e^{-\left(39.47^{*} \tau\right)}\right)\left(\sin \left[2 \pi\left(\frac{y}{h}+1\right)\right]\right), \\
& \frac{u_{t, 5}}{U}=\left[(-0.1272)+\left(\frac{4 \Omega_{E} E_{x}}{U}\right)(0.0551)\right]\left(e^{-(61.5 * \tau)}\right)\left(\sin \left[\frac{5 \pi}{2}\left(\frac{y}{h}+1\right)\right]\right), \\
& \frac{u_{t, 6}}{U}=[(0.1061)]\left(e^{-\left(88.8^{*} \tau\right)}\right)\left(\sin \left[3 \pi\left(\frac{y}{h}+1\right)\right]\right) .
\end{aligned}
$$

\section{Steady state analysis for heat transfer and entropy generation}

Once the velocity distributions are known, the temperature distributions for the two regions are determined by solving the steady state heat transfer energy equation with appropriate boundary conditions. In the present problem, it is assumed that the two walls are maintained at constant temperatures $T_{1}$ and $T_{2}$. The governing equation for the steady state heat transfer is given by

$$
\alpha \frac{d^{2} T}{d y^{2}}+\frac{\mu}{\rho c_{p}}\left(\frac{\partial u_{s}}{\partial y}\right)^{2}=0 .
$$

With the aid of transformation $Y=y+h$ the above equation will become

$$
\frac{d^{2} T}{d Y^{2}}+\frac{\operatorname{Pr}}{c_{p}}\left(\frac{\partial u_{s}}{\partial Y}\right)^{2}=0
$$

where $\operatorname{Pr}=\frac{v}{\alpha}$ the Prandtl number. 
The appropriate boundary conditions are considered as

$$
\begin{aligned}
& T=T_{1} \quad \text { at } \quad Y=0, \\
& T=T_{2} \quad \text { at } \quad Y=2 h .
\end{aligned}
$$

Define the similarity transformations as

$$
\bar{u}=\frac{u_{s}}{U}, \quad \theta=\frac{T-T_{1}}{T_{2}-T_{1}} .
$$

Therefore, using these transformations in Eq.(3.1) one can get

$$
\frac{d^{2} \theta}{d Y^{2}}+\operatorname{Br}\left(\frac{\partial \bar{u}}{\partial Y}\right)^{2}=0
$$

where $\mathrm{E}_{\mathrm{c}}=\frac{U^{2}}{c_{p}\left(T_{2}-T_{1}\right)}$ is the Eckert number and $\operatorname{PrE}_{\mathrm{c}}=\mathrm{Br}$ is the Brinkman number.

Corresponding boundary conditions are

$$
\begin{array}{lll}
\theta=0 \quad \text { at } & Y=0, \\
\theta=1 \quad \text { at } & Y=2 h .
\end{array}
$$

\section{Second law analysis}

According to Bejan (1996), the rate of entropy generation that arises due to heat transfer and fluid friction losses can be derived as

$$
S_{g}^{\prime \prime \prime}=\frac{k}{T_{0}^{2}}\left(\frac{\partial T}{\partial Y}\right)^{2}+\frac{\mu}{T_{0}}\left(\frac{\partial u_{s}}{\partial Y}\right)^{2}
$$

where $T_{0}$ is the absolute reference temperature.

Define similarity variable as $\eta=\frac{Y}{2 h}$. Using this variable and Eqs (3.3) one can obtain the dimensionless entropy generation equation as

$$
N_{s}=\left(\frac{\partial \theta}{\partial \eta}\right)^{2}+\frac{B r}{\Omega}\left(\frac{\partial \bar{u}}{\partial \eta}\right)^{2}
$$

where $\quad \mathrm{Br}=\frac{\mu}{k} \frac{U^{2}}{\left(T_{2}-T_{1}\right)}, \Omega^{-1}=\frac{T_{0}}{\left(T_{2}-T_{1}\right)}$ and $N_{s}=\frac{T_{0}^{2} * 4 h^{2}}{k\left(T_{2}-T_{1}\right)^{2}} S_{g}^{\prime \prime \prime}$.

In Eq.(4.1)

$$
\left(\frac{\partial \theta}{\partial \eta}\right)^{2}=N_{H}=\text { entropy due to heat transfer, }
$$




$$
\begin{aligned}
& \frac{\mathrm{Br}}{\Omega_{T}}\left(\frac{\partial \bar{u}}{\partial \eta}\right)^{2}=N_{F}=\text { entropy due to fluid friction, } \\
& \therefore N_{s}=N_{H}+N_{F} .
\end{aligned}
$$

The irreversibility ratio is defined as the ratio of entropy generation due to fluid friction to the entropy due to heat transfer, so is given by

$$
\phi=\frac{N_{F}}{N_{H}} .
$$

The Bejan number is defined as the ratio of entropy generation due to heat transfer to the total entropy generation.

$$
\mathrm{Be}=\frac{N_{H}}{N_{H}+N_{F}}=\frac{1}{1+\phi} .
$$

Solving Eq.(3.4) one can get

$$
\begin{aligned}
& \theta(y)=(-\mathrm{Br})\left[\frac{y^{2}}{8 h^{2}}+\left(\frac{\Omega_{E} E_{x}}{U}\right)^{2}\left[\frac{2 \cos h^{2}\left(k_{w} y\right)-\left(1+2 k_{w}^{2} y^{2}\right)}{8 \cdot \cos h^{2}\left(k_{w} h\right)}\right]+\left(\frac{\Omega_{E} E_{x}}{U}\right) \frac{\sinh \left(k_{w} y\right)}{\left(k_{w} h\right) \cosh \left(k_{w} h\right)}+\right. \\
& -\left[\frac{1}{(2 \cdot \mathrm{Br} \cdot h)}+\left(\frac{\Omega_{E} E_{x}}{U}\right) \frac{\tanh \left(k_{w} h\right)}{k_{w} h^{2}}\right] y-\left[\frac{1}{(2 \cdot \mathrm{Br})}+\frac{1}{8}+\left(\frac{\Omega_{E} E_{x}}{U}\right)^{2}\left[\frac{2 \cos h^{2}\left(k_{w} h\right)-\left(1+2 k_{w}^{2} h^{2}\right)}{8 \cdot \cos h^{2}\left(k_{w} h\right)}\right]\right], \\
& \left(\frac{\partial \theta}{\partial y}\right)^{2}=\left[( - \mathrm { Br } ) \left[\frac{y}{4 h^{2}}+\left(\frac{\Omega_{E} E_{x}}{U}\right)^{2}\left[\frac{\left(4 \mathrm{k}_{\mathrm{w}}\right) \sinh \left(k_{w} y\right) \cosh \left(k_{w} y\right)-\left(4 k_{w}^{2} y\right)}{8 \cos h^{2}\left(k_{w} h\right)}\right]+\right.\right. \\
& \left.+\left(\frac{\Omega_{E} E_{x}}{U}\right) \frac{\cosh \left(k_{w} y\right)}{(h) \cosh \left(k_{w} h\right)}-\left[\frac{1}{(2 \mathrm{Br} h)}+\left(\frac{\Omega_{E} E_{x}}{U}\right) \frac{\tanh \left(k_{w} h\right)}{k_{w} h^{2}}\right]\right]^{2}=N_{H}, \\
& \frac{\operatorname{Br}}{\Omega}\left(\frac{\partial \bar{u}}{\partial y}\right)^{2}=\left[\frac{1}{(2 h)}+\left(\frac{\Omega_{E} E_{x}}{U}\right)\left(k_{w}\right)\left(\frac{\sinh \left(k_{w} y\right)}{\cosh \left(k_{w} h\right)}\right)\right]^{2} * \frac{\mathrm{Br}}{\Omega}=N_{F} .
\end{aligned}
$$

\section{Results and discussion}

The velocity distribution $u_{t}$ and $u_{s}$ and the temperature distributions are obtained analytically from the momentum equation and energy equation using separation of variable method. The effects of the dimensionless entropy generation number, Bejan number, irreversibility ratio, entropy generation due to fluid friction, entropy generation due to heat transfer on dimensionless time, relative channel height, Brinkman number, dimensionless temperature ratio, nanoparticle volume fraction are presented.

Figure 2 shows the steady state velocity distribution for various values of volume fraction of nanoparticle. We can observe that the steady state velocity increases for increasing values of nanoparticle volume fraction. 


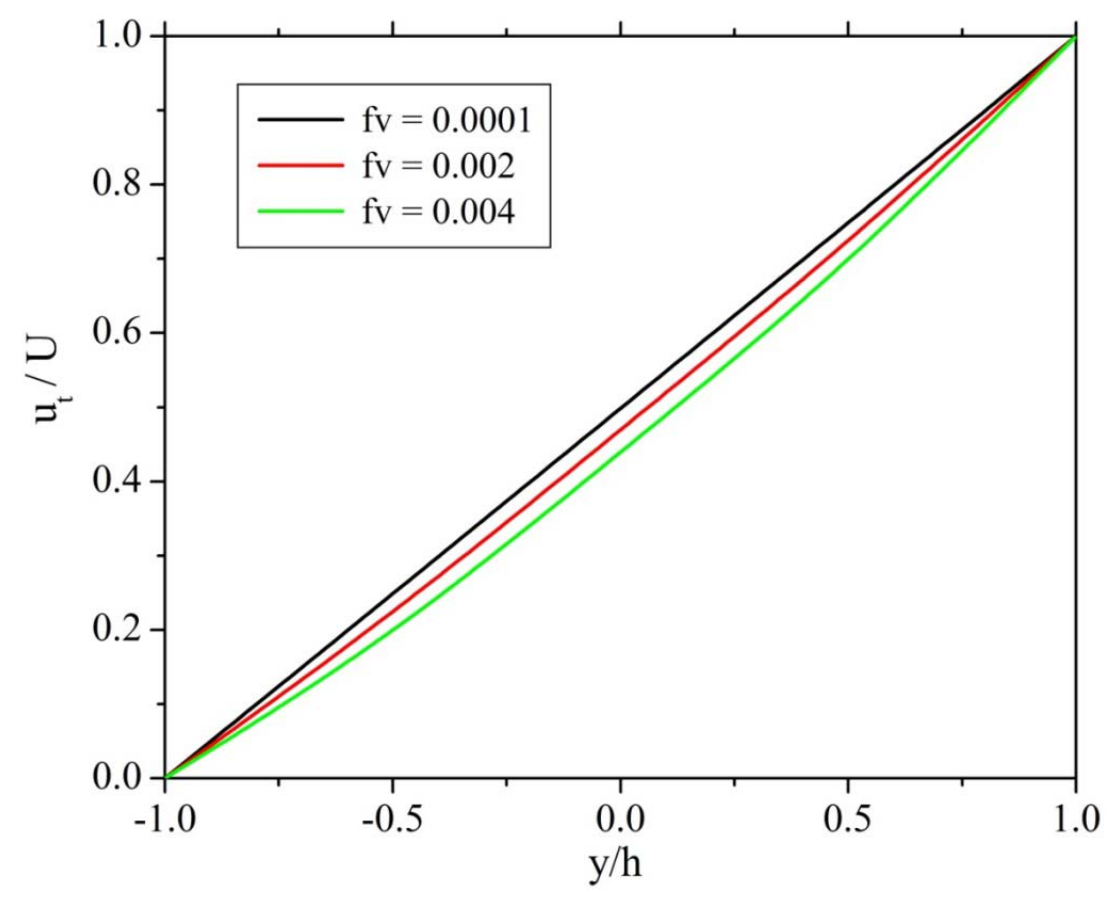

Fig.2. Steady state velocity profiles for increasing values of nanoparticle volume fraction with $r_{p}=5 \mathrm{~nm}$.

Figures 3, 4 and 5depict the transient velocity (absolute) distribution at various points in the flow for different values of nanoparticle volume fraction. From these figures, it is observed that as the value of $\boldsymbol{\tau}$ (dimensionless time) increases, the transient velocity decreases i.e., the flow approaches steady state. Further one can see that the transient velocity is maximum inbetween $y / h=0$ and $y / h=0.5$.

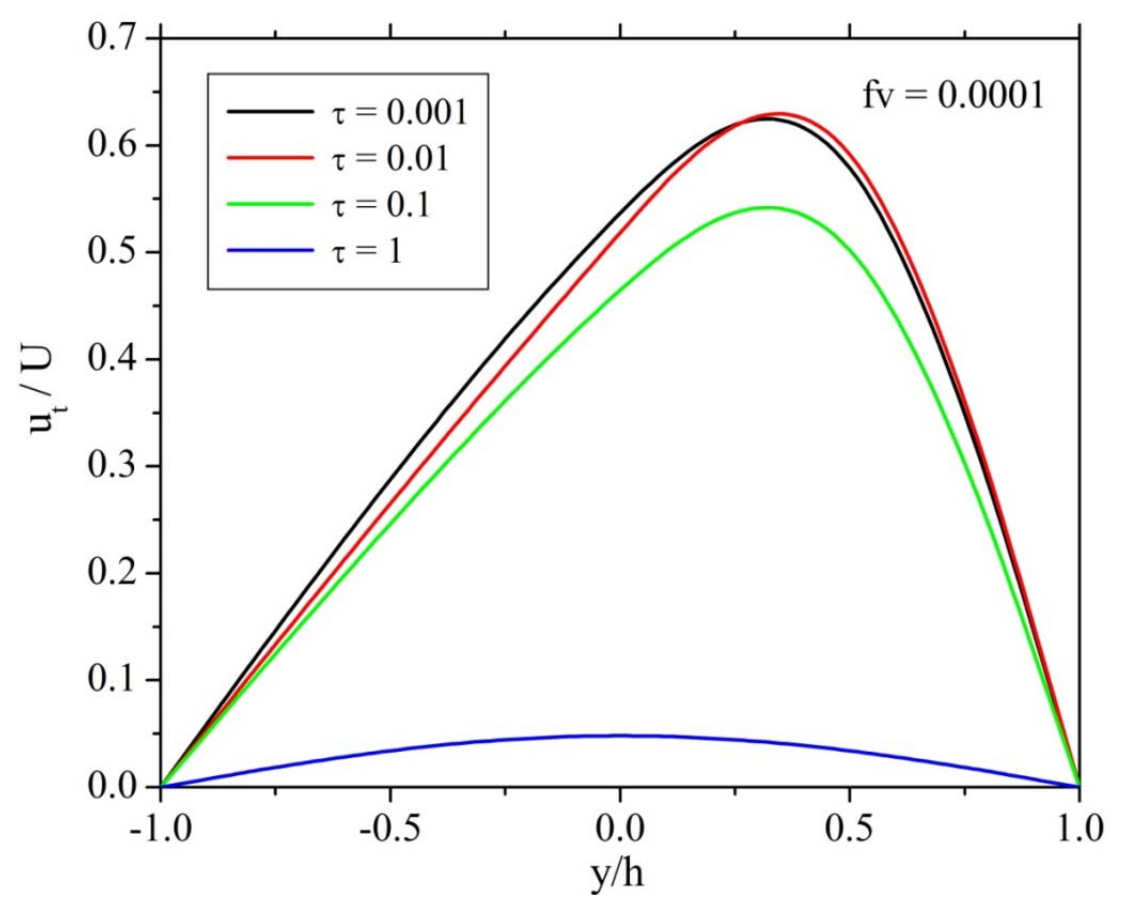

Fig.3. Transient velocity profiles for increasing values of dimensionless time in $e^{-\left(\frac{\boldsymbol{n}^{2} \pi^{2}}{4 \boldsymbol{h}^{2}} \boldsymbol{v} \boldsymbol{t}\right)}$. 


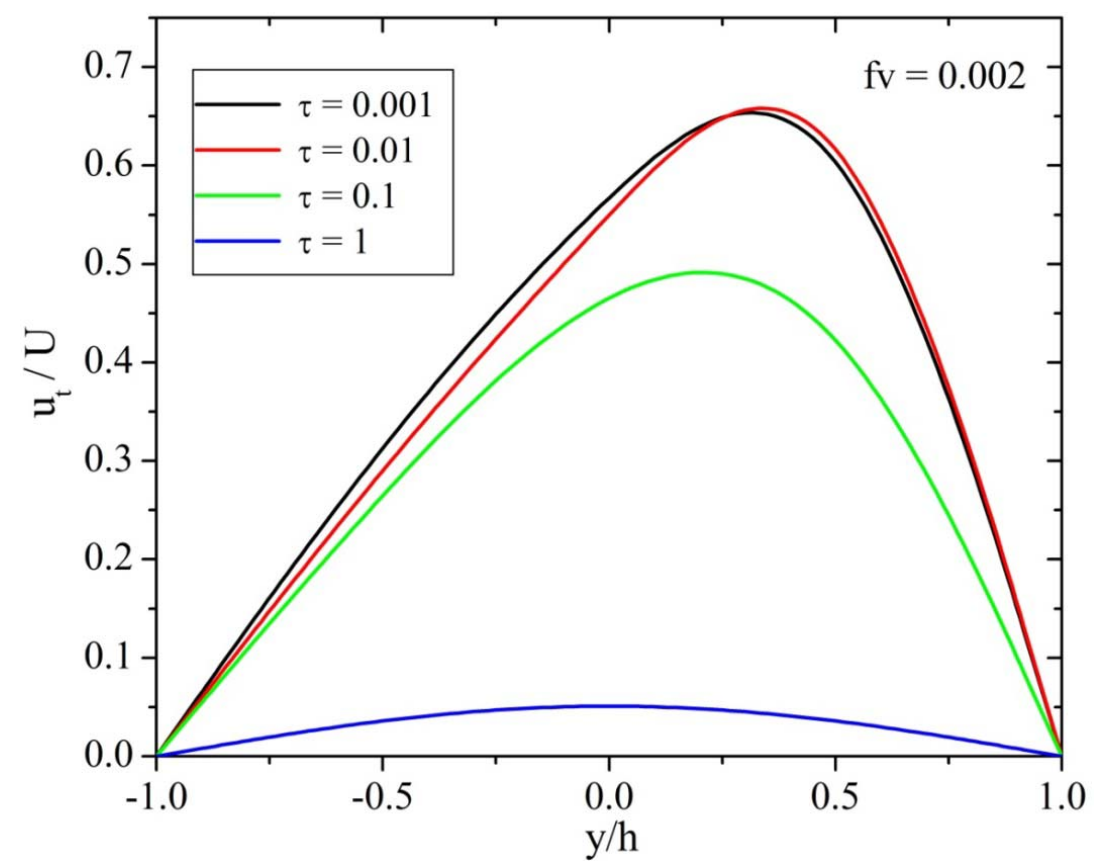

Fig.4. Transient velocity profiles for increasing values of dimensionless time in $e^{-\left(\frac{n^{2} \pi^{2}}{4 \boldsymbol{h}^{2}} * v t\right)}$.

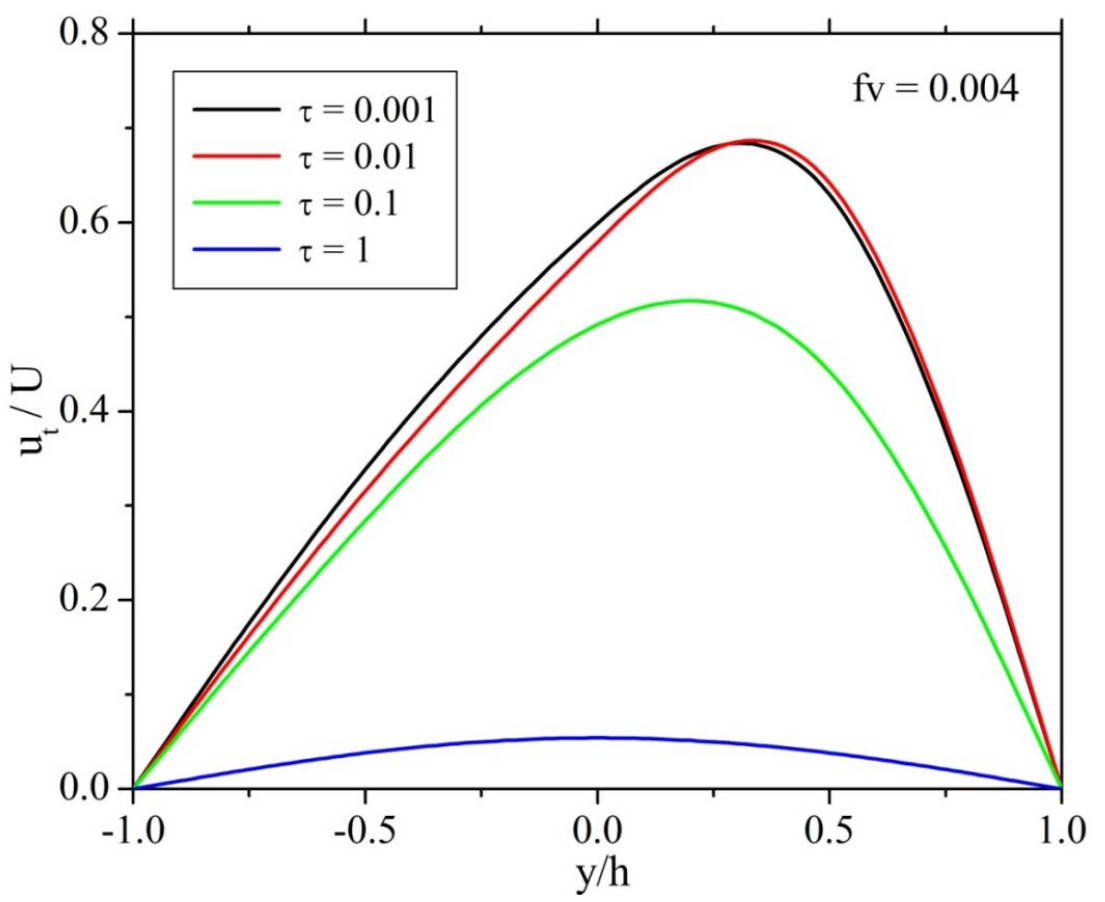

Fig.5. Transient velocity profiles for increasing values of dimensionless time in $e^{-\left(\frac{n^{2} \pi^{2}}{4 h^{2}} * v t\right)}$.

Figures 6, 7, 8, 9 and 10 are plotted for constant values of nanoparticle volume fraction and $\Omega$ for various values of the Brinkman number to analyze its effect on entropy due to heat transfer, entropy due to fluid friction, irreversibility ratio and Bejan number. It can be seen from these plots that as the Brinkman number increases, entropy due to heat transfer, entropy due to fluid friction and total entropy increase but an opposite 
phenomenon can be found as the width of the channel increases. For the irreversibility ratio, as $\mathrm{Br}$ increases $\Phi$ also increases and it attains the highest value at $y / h=1$. For some larger values of $\mathrm{Br}$ the Bejan number decreases with an increase in distance and also the distribution is relatively flat for smaller values of the Brinkman number.

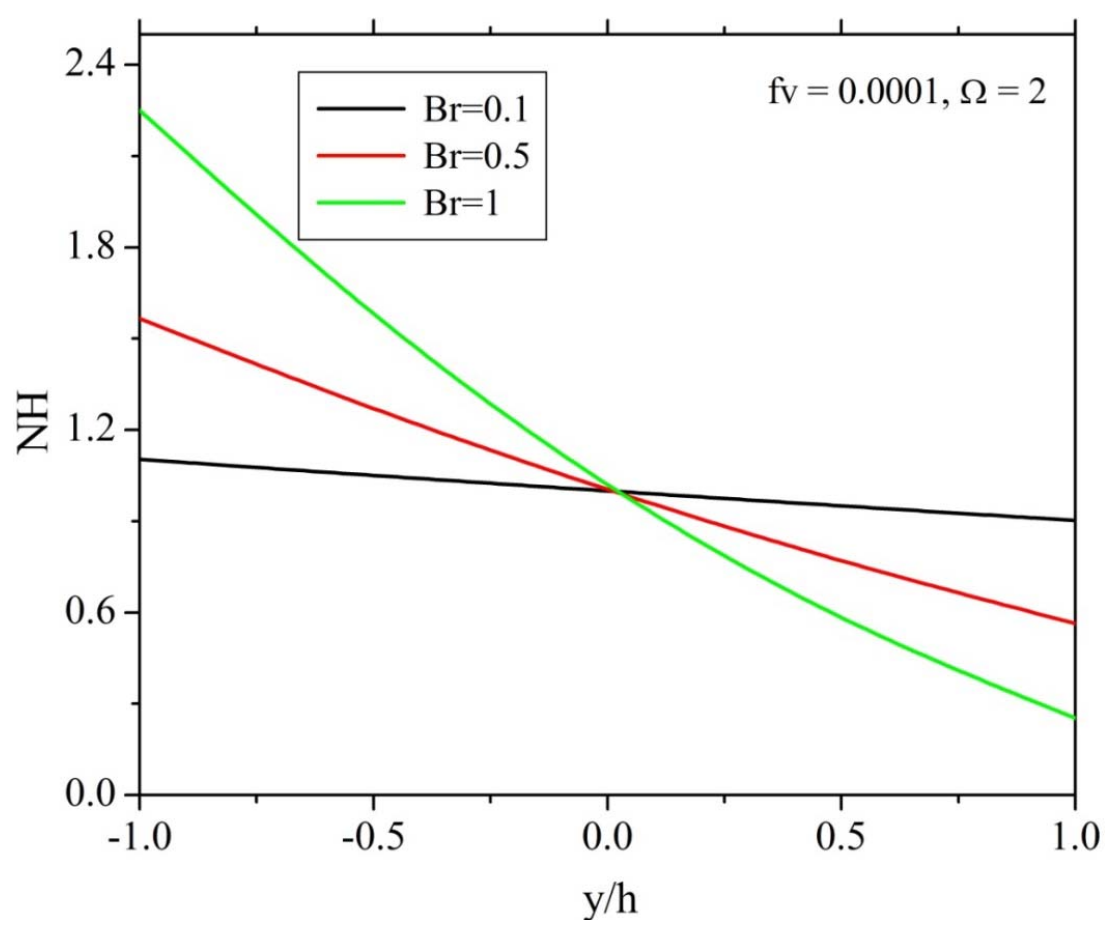

Fig.6. Entropy due to heat transfer profiles for increasing values of the Brinkman number.

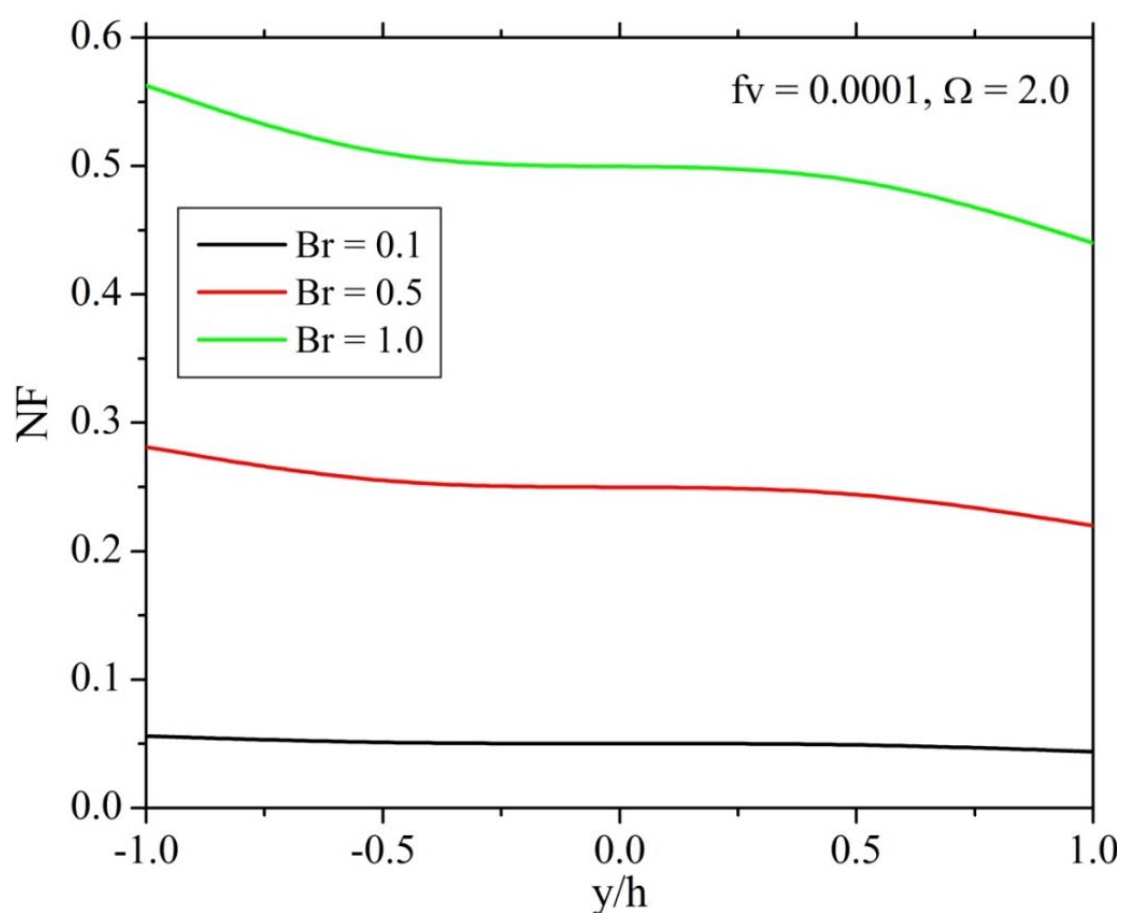

Fig.7. Entropy due to fluid friction profiles for increasing values of the Brinkman number. 


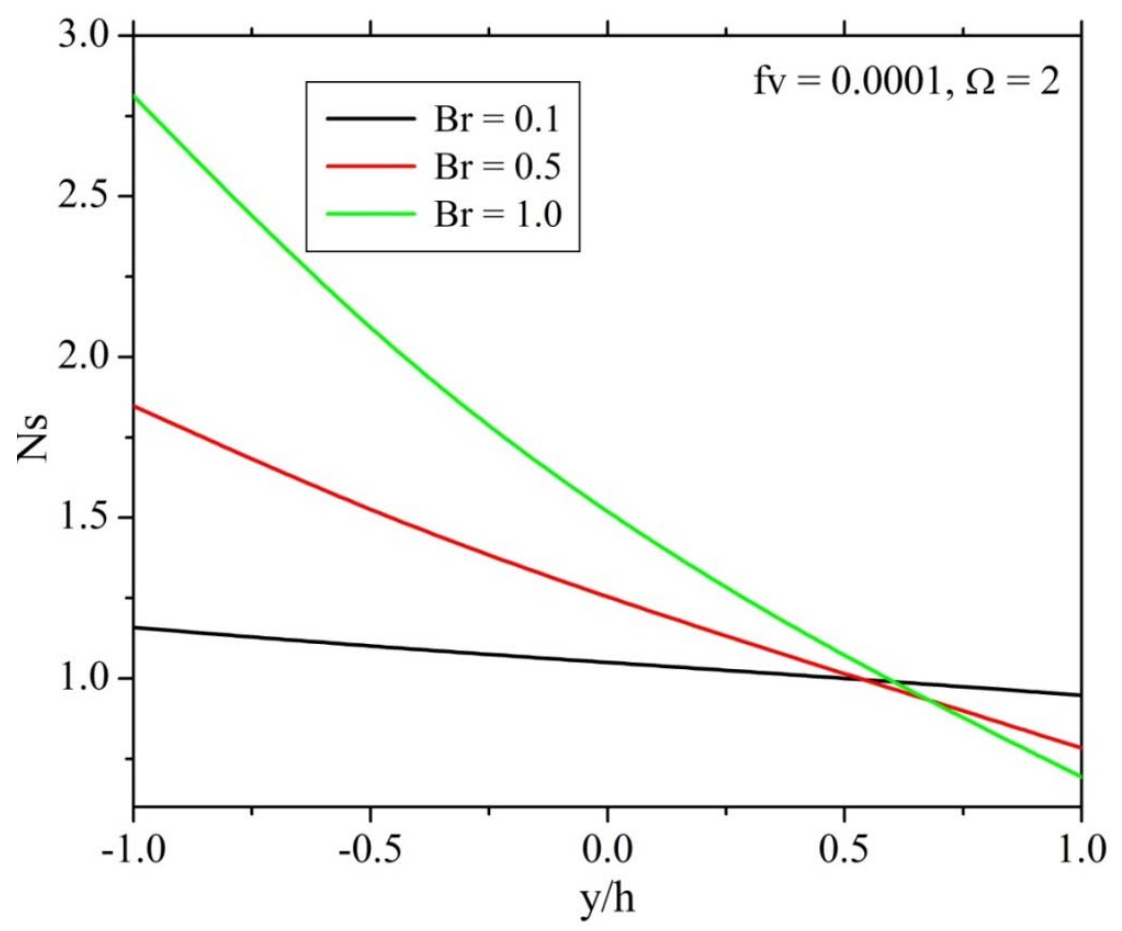

Fig.8.Total entropy generation $N_{s}=N_{H}+N_{F}$ for increasing values of the Brinkman number.

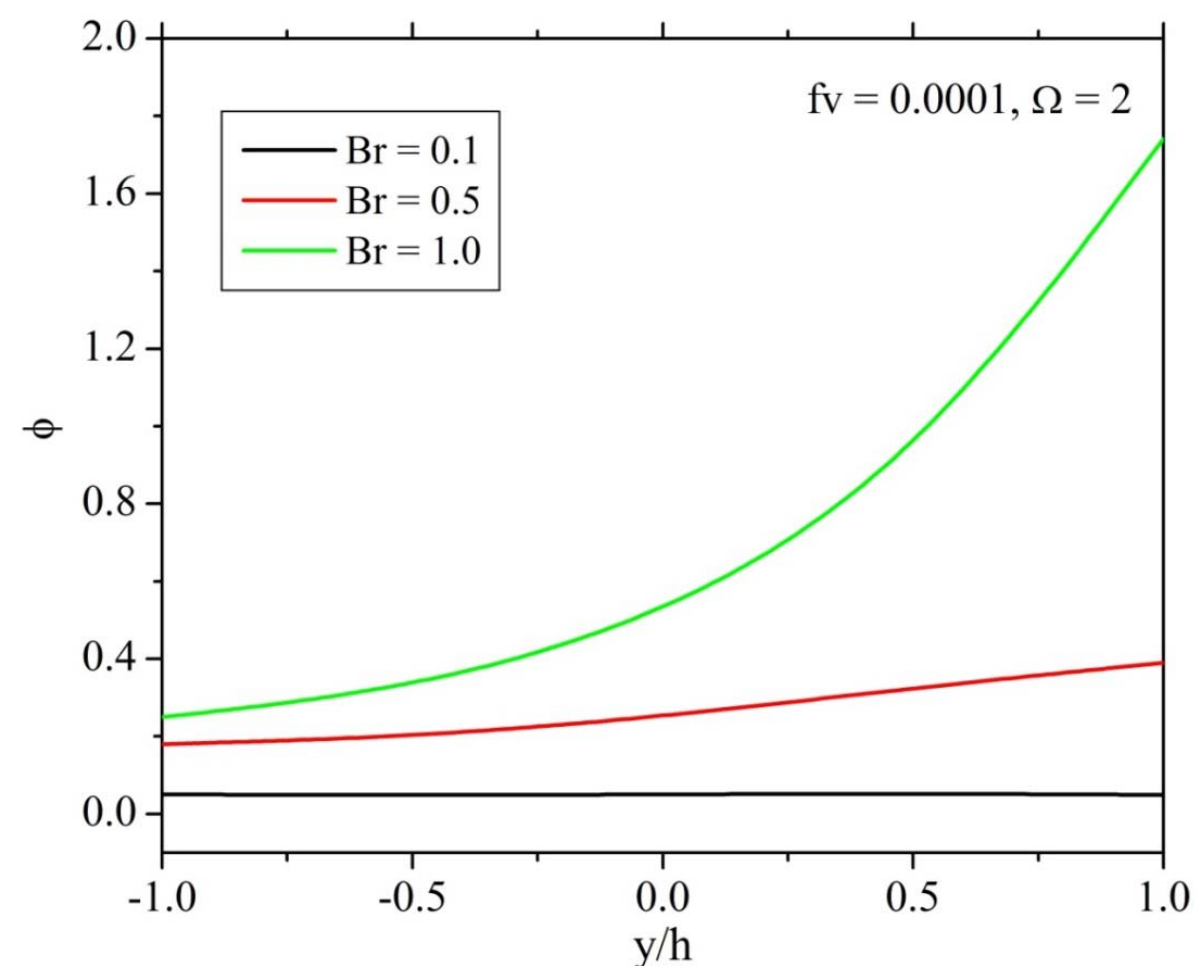

Fig.9. Irreversibility ratio profiles for increasing values of the Brinkman number. 


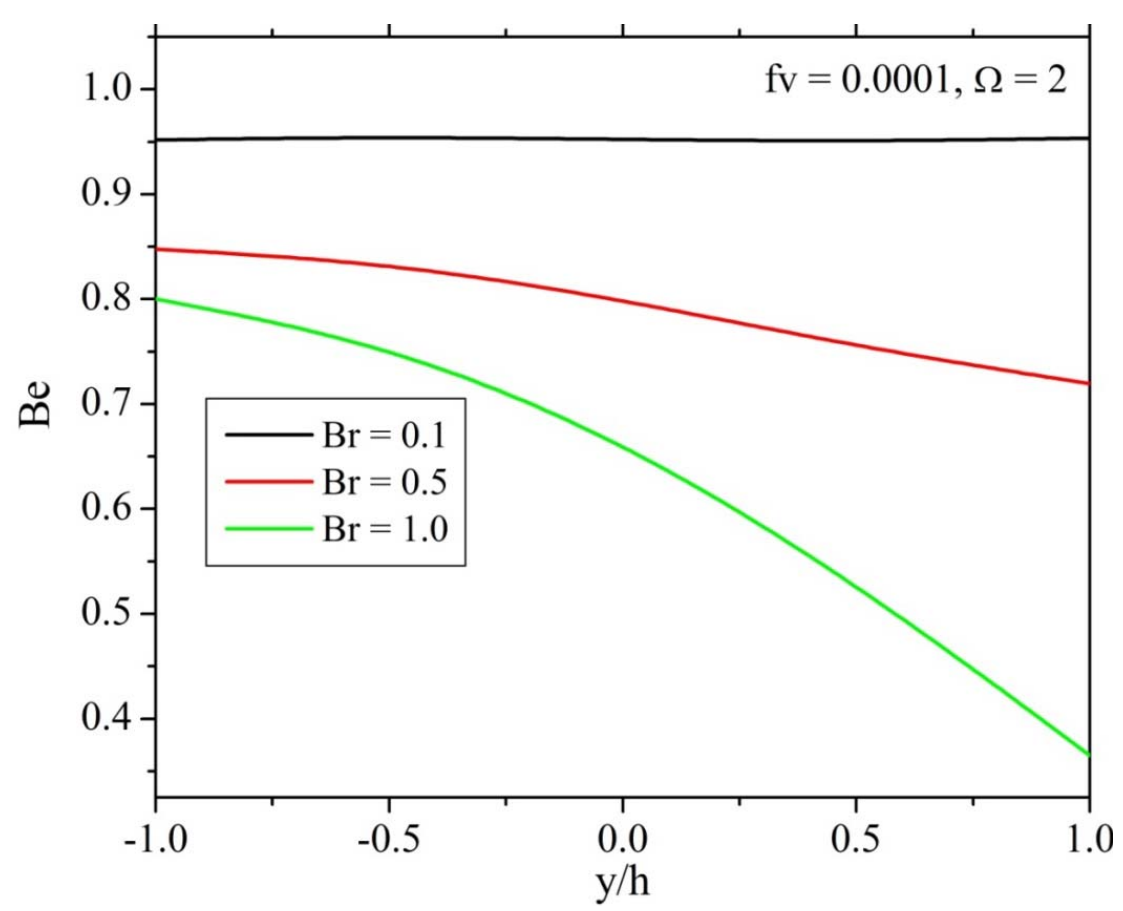

Fig.10. Variation of the Bejan number for increasing values of the Brinkman number.

Figures 11, 12,13, 14 and 15 are drawn for a constant Brinkman number and $\Omega$ with different values of nanoparticle volume fraction. As the values of nanoparticle volume fraction increase, entropy due to heat transfer, entropy due to fluid friction and total entropy also increase but it decreases with distance. As nanoparticle volume fraction increases the irreversibility $\Phi$ decreases with distance and for higher nanoparticle volume fraction, the distributions become more parabolic with the lowest value occurring at the lower plate. As $f_{v}$ increases the Bejan number increases and it becomes more parabolic with the highest value occurring at $y / h=0$.

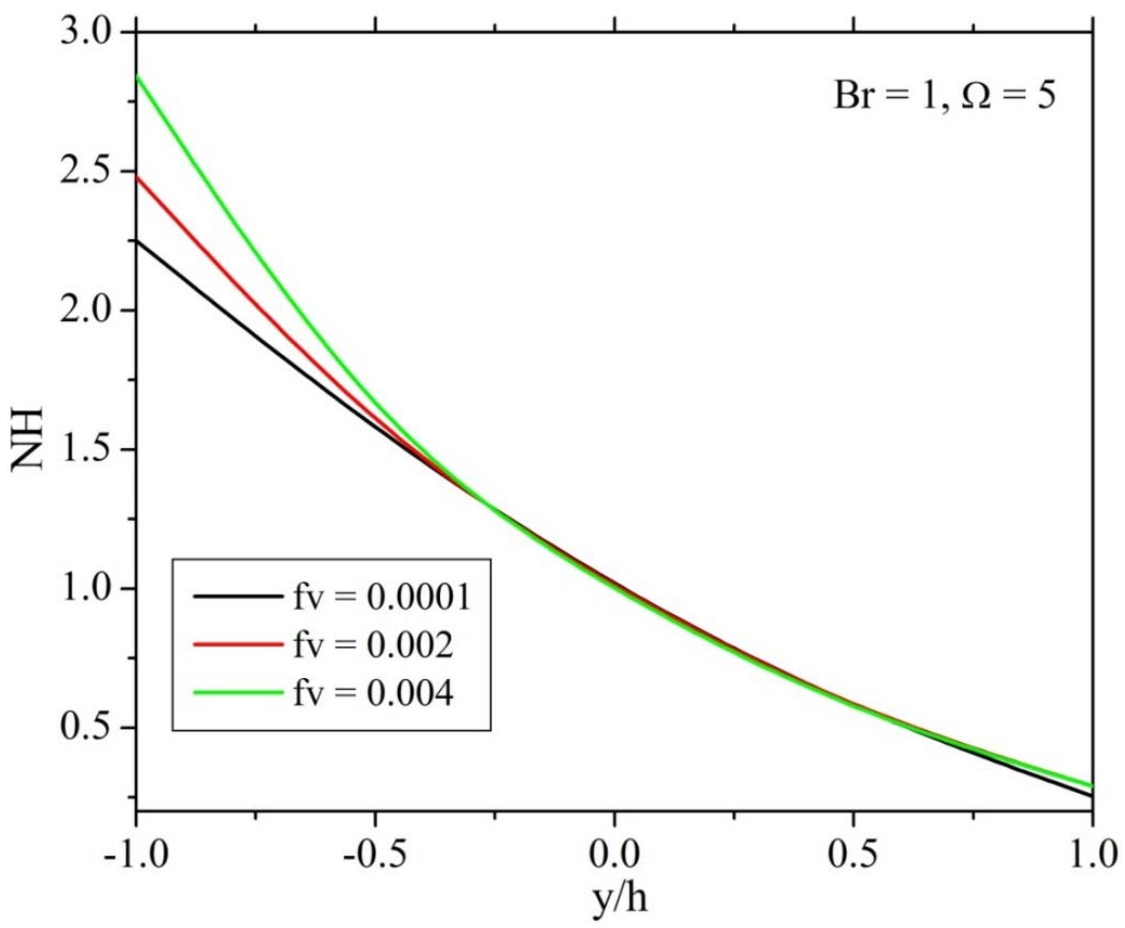

Fig.11. Entropy due to heat transfer profiles for increasing values of nanoparticle volume fraction. 


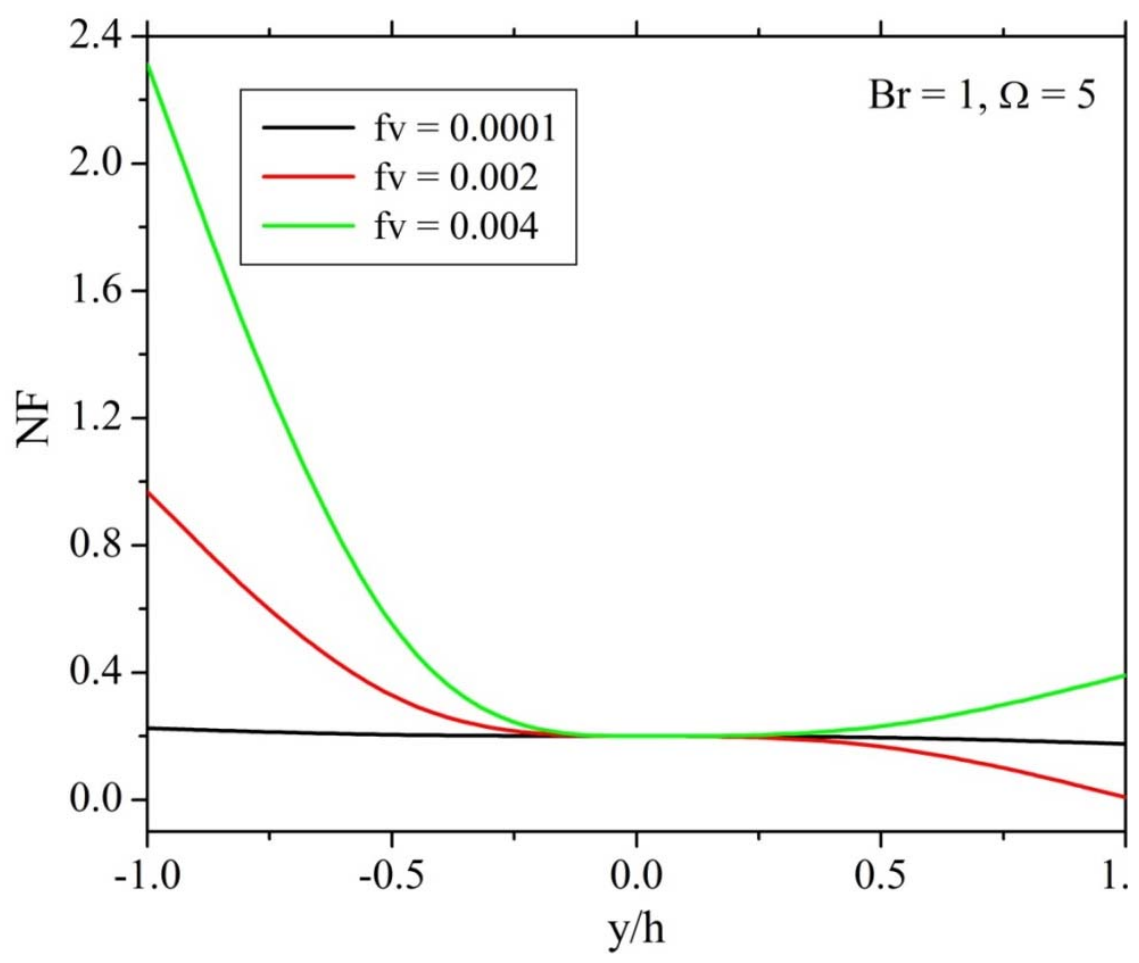

Fig.12. Entropy due to fluid friction profiles for increasing values of nanoparticle volume fraction.

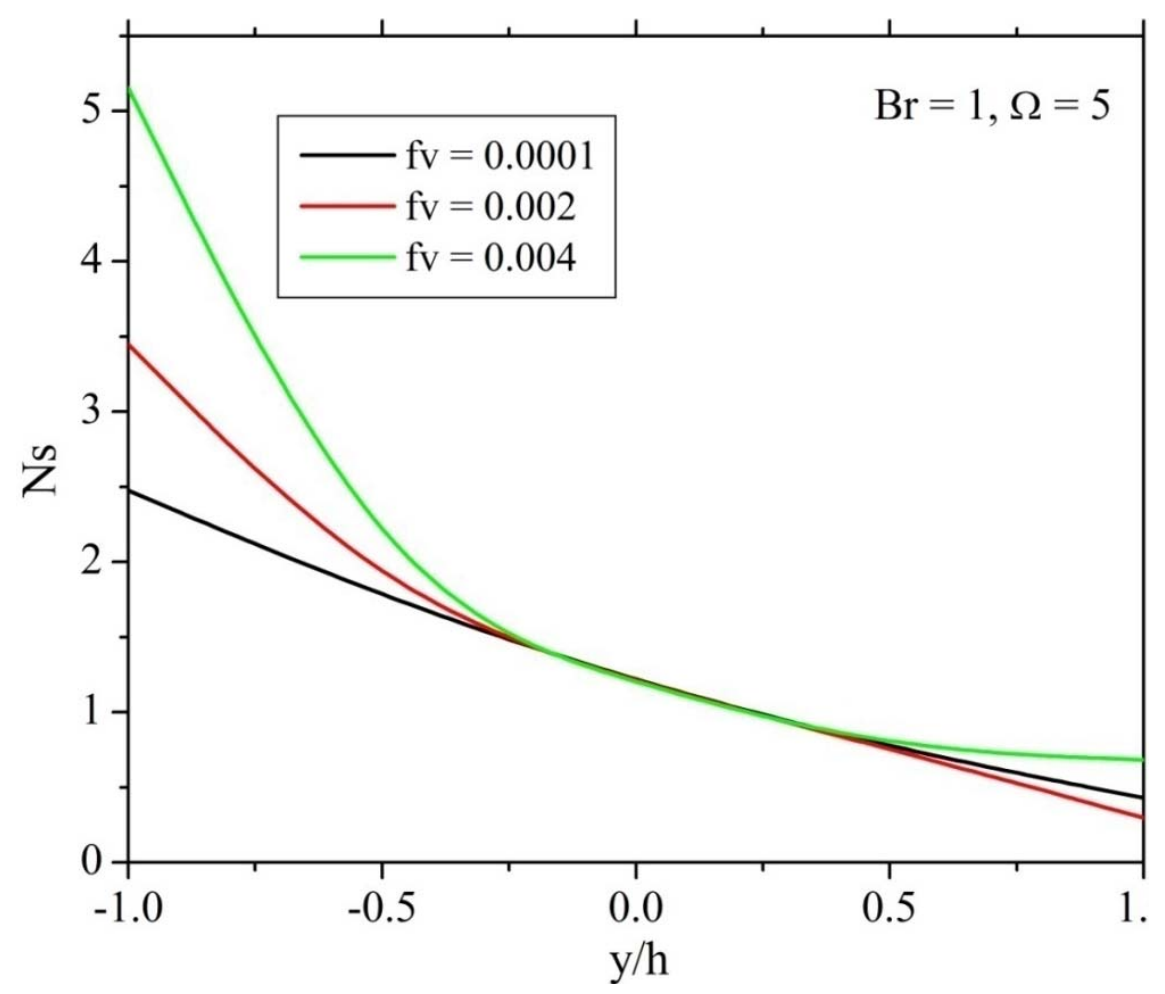

Fig.13. Total entropy generation for increasing values of nanoparticle volume fraction. 


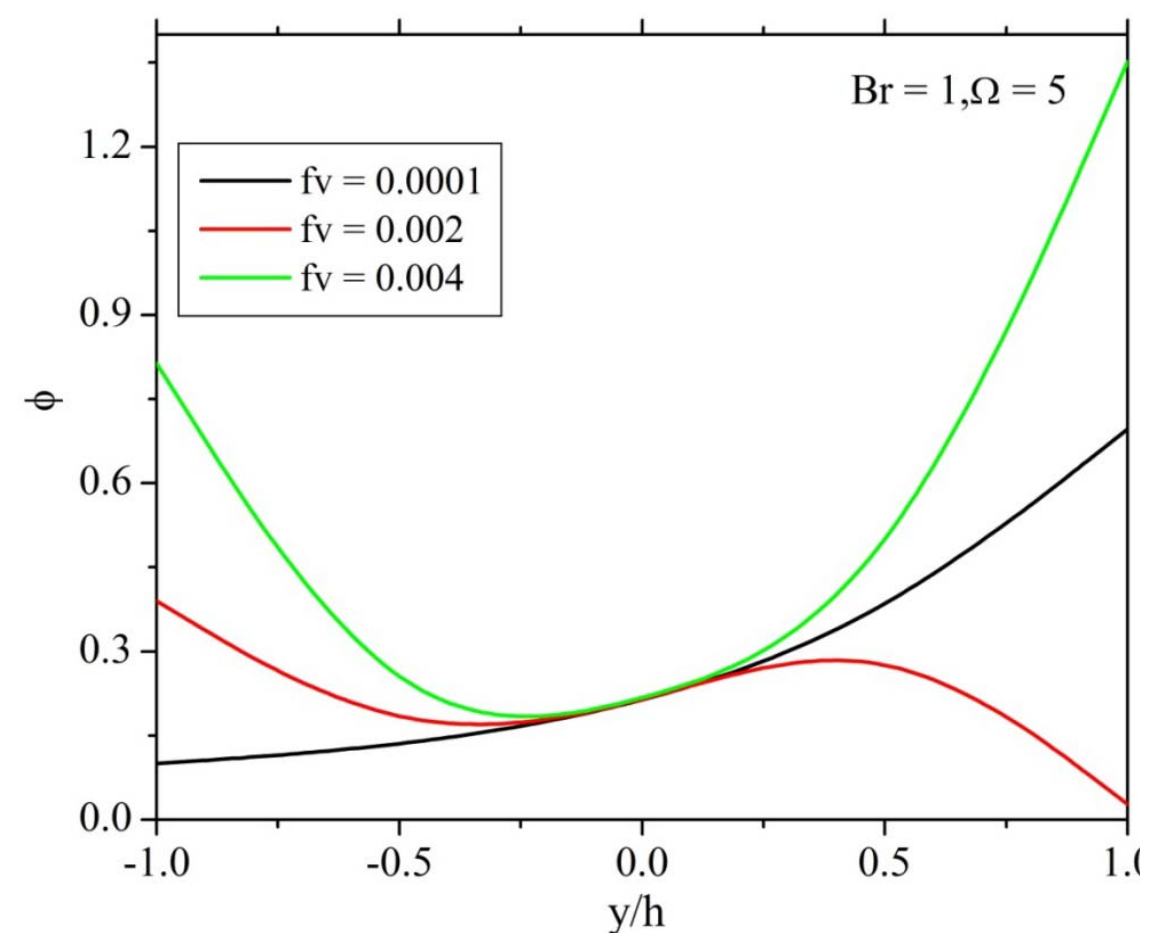

Fig.14. Irreversibility ratio profiles for increasing values of nanoparticle volume fraction.

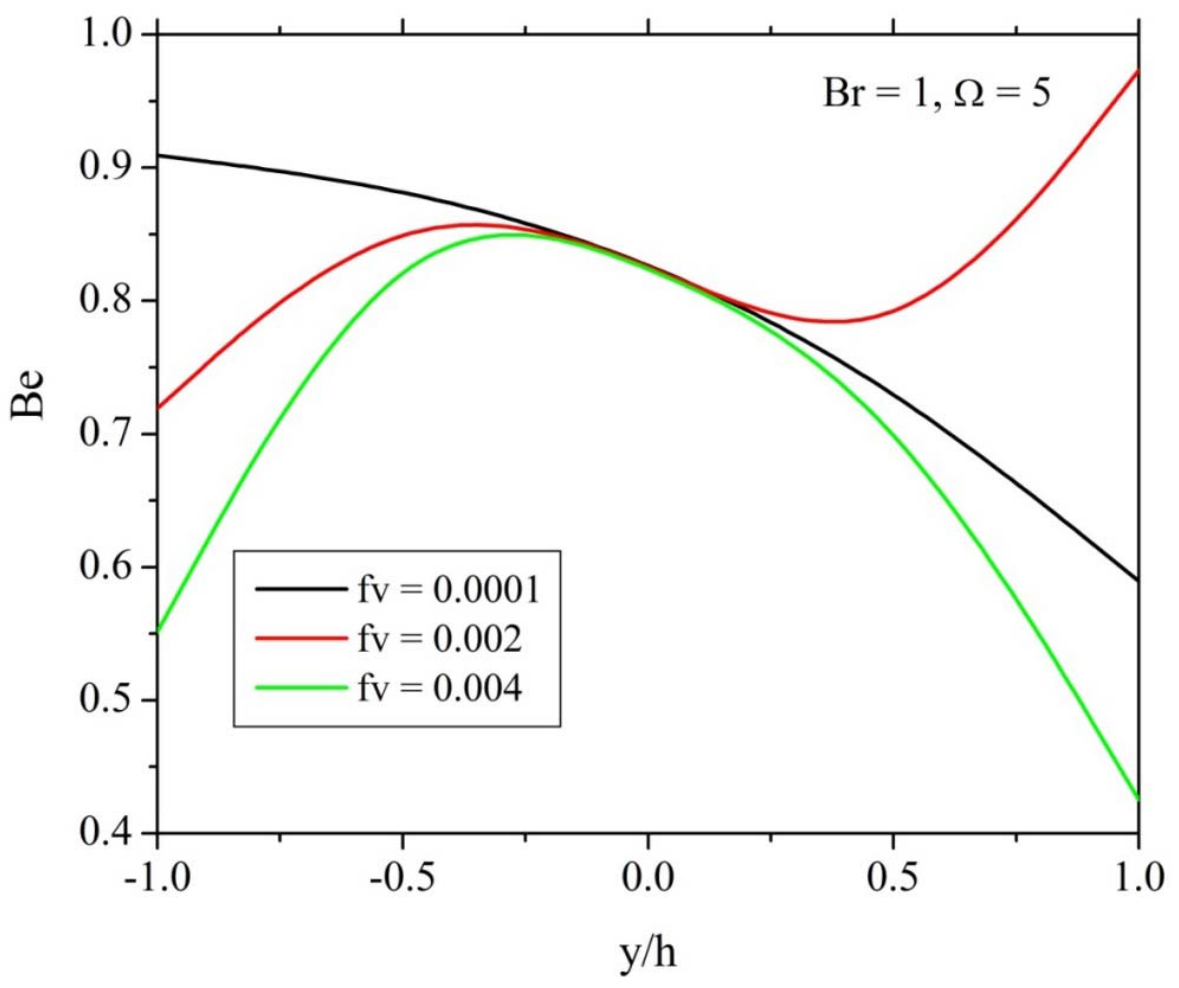

Fig.15. Variation of the Bejan number for increasing values of nanoparticle volume fraction.

Figures 16, 17, 18 and 19 show entropy generation numbers, the Bejan number and irreversibility for constant values of nanoparticle volume fraction, Brinkman number by varying $\Omega$ for entropy due to heat 
transfer and entropy due to fluid friction. As $\Omega$ increases, both entropy due to heat transfer and entropy due to fluid friction decreases. Further the entropy due to heat transfer, and entropy due to fluid friction decreases with an increase in distance. Also, one can see that the Bejan number increases with an increase in $\Omega$ whereas it decreases with distance. Final observation shows that $\phi$ decreases, as $\Omega$ increases and it increases with distance.

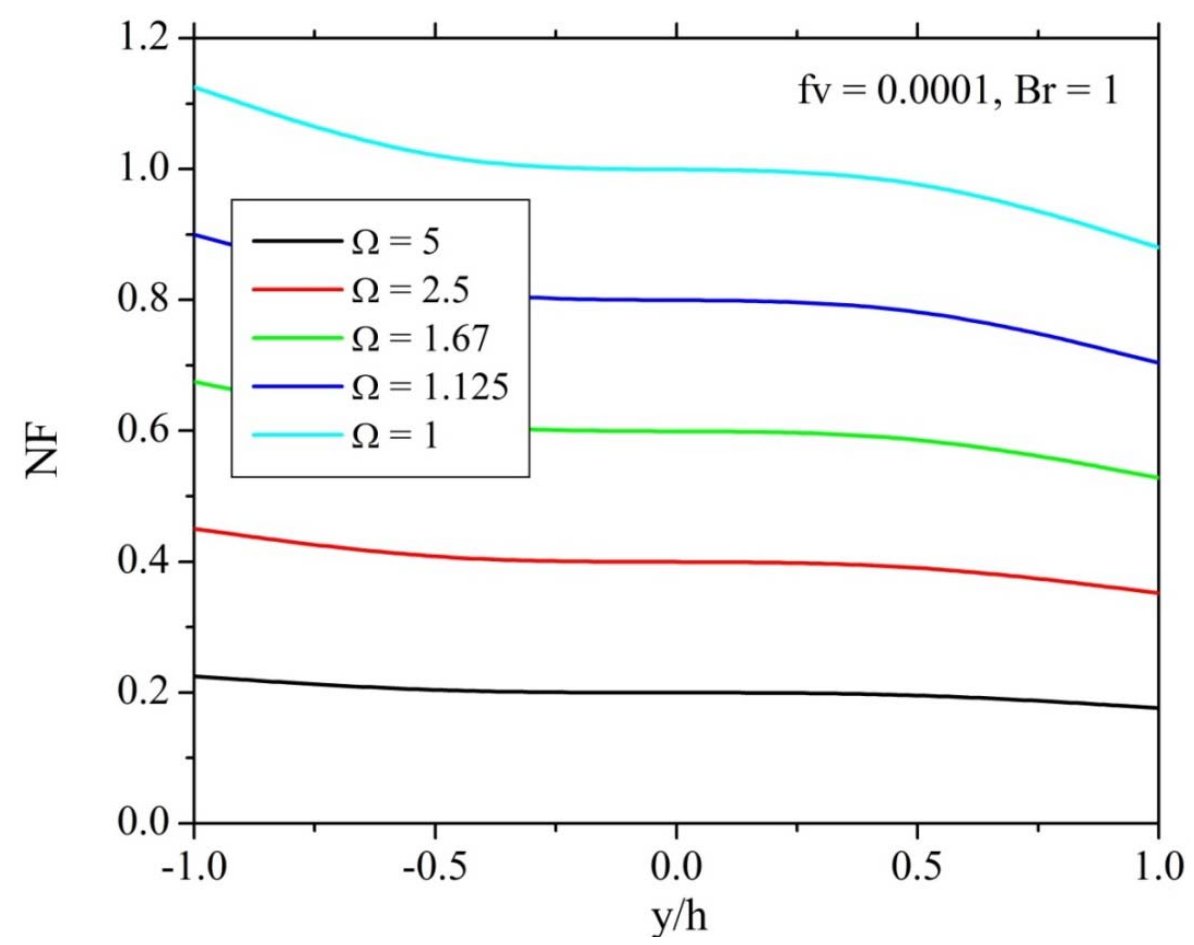

Fig.16. Entropy due to fluid friction profiles for increasing values of $\Omega$.

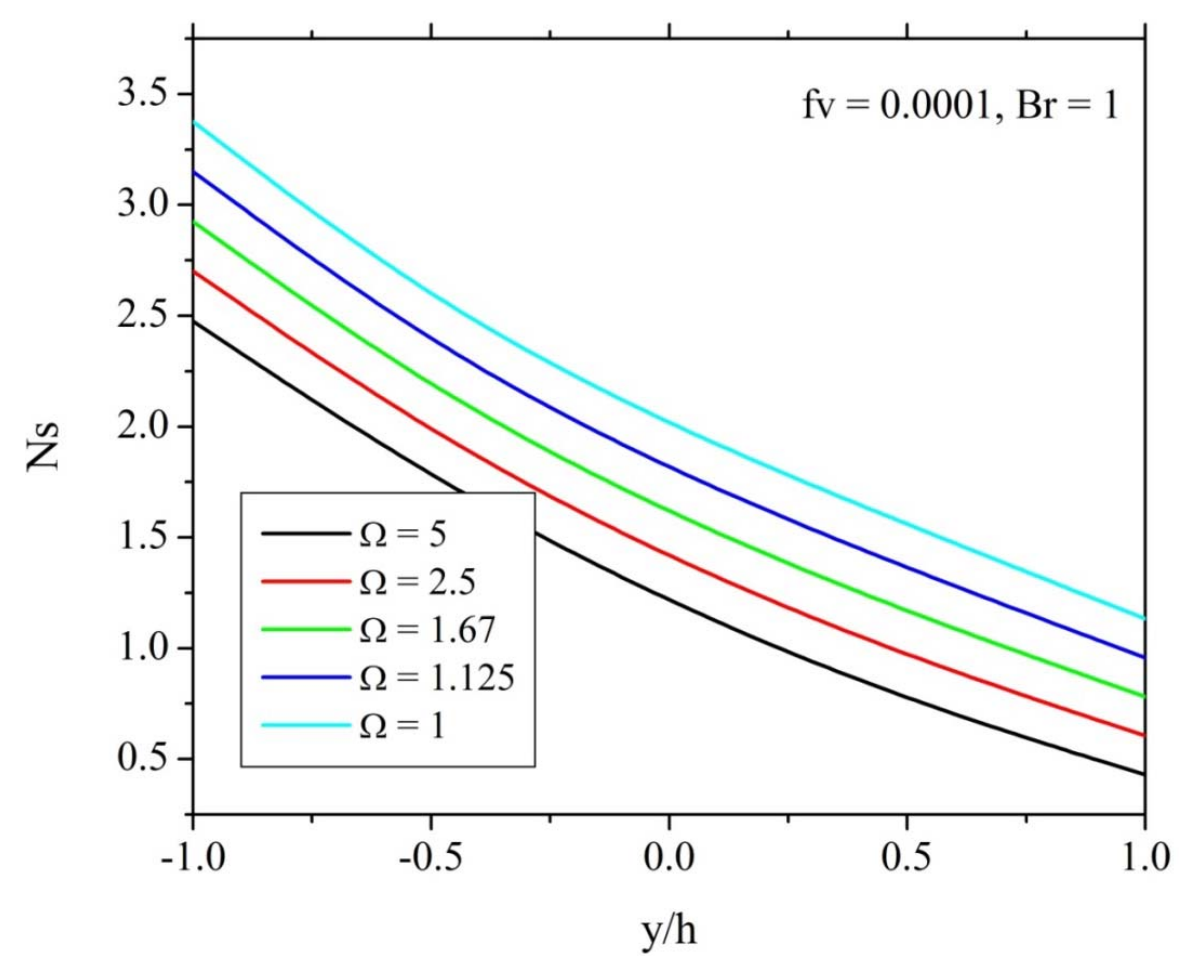

Fig.17. Total entropy profiles for increasing values of $\Omega$. 


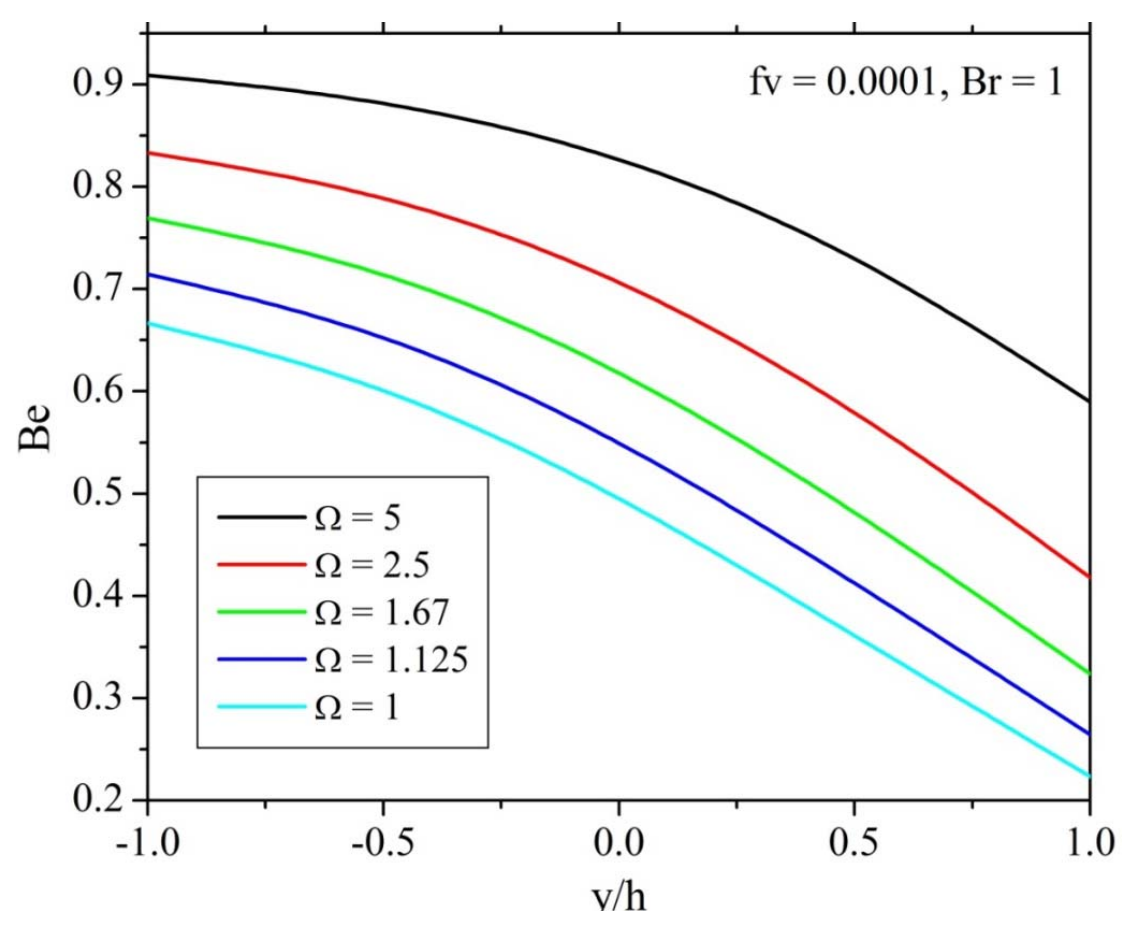

Fig.18. Variation of the Bejan number for increasing values of $\Omega$.

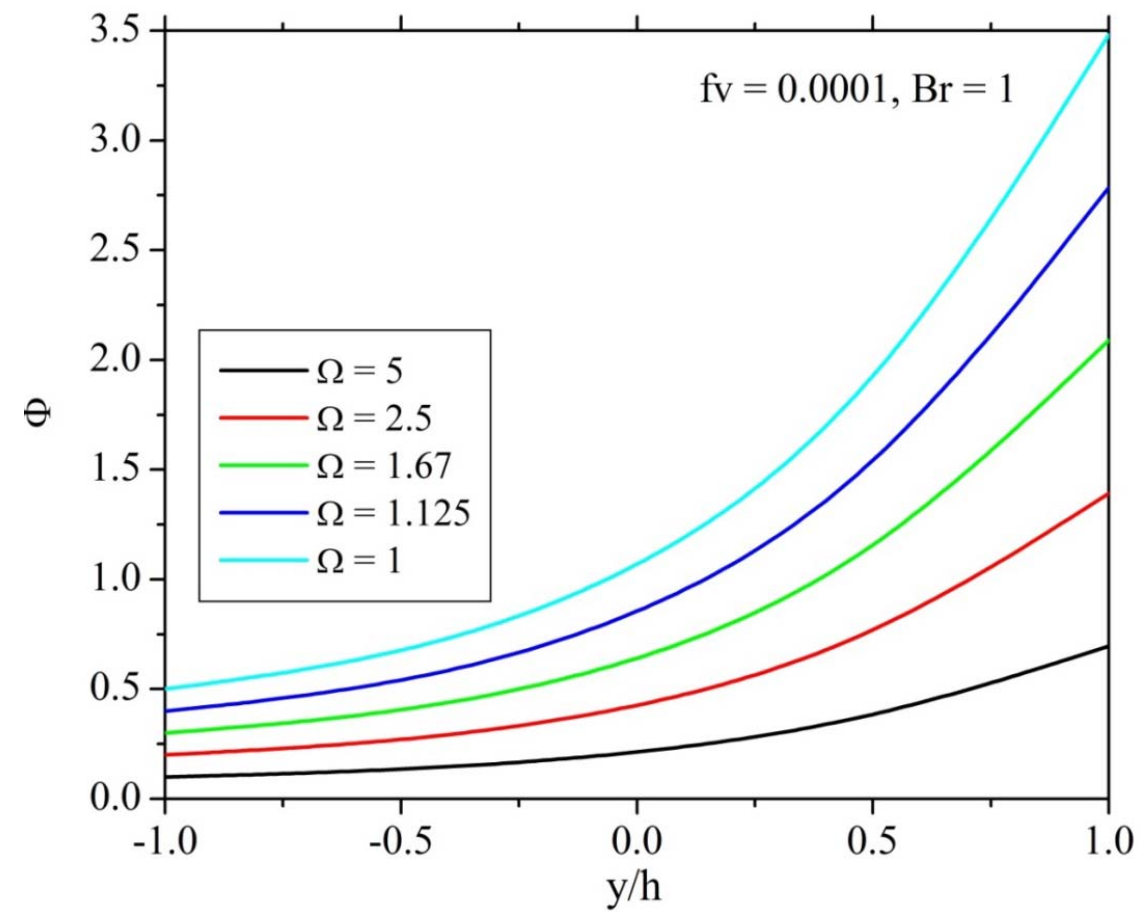

Fig.19. Irreversibility ratio profiles for increasing values of $\Omega$.

\section{Concluding remarks}

A fully developed Couette flow with heat transfer is considered under the influence of voltage potential gradient along the length of a channel. An analysis for the transient velocity, steady state velocity and entropy generated in a parallel plate micro-channel has been made. 
The main outcomes of the problem are summarized as follows:

- The steady state velocity increases for increasing values of nanoparticle volume fraction.

- The transient velocity decreases as the value of dimensionless time increases and is maximum inbetween $y / h=0$ and $y / h=0.5$.

- The entropy due to heat transfer, entropy due to fluid friction and total entropy increase with the Brinkman number, but an opposite phenomenon can be found as the width of the channel increases. The same effect can be found with nanoparticle volume fraction.

- The irreversibility ratio increases as the Brinkman number $\mathrm{Br}$ increases and it attains a maximum value at $y / h=1$. An opposite effect can be seen under the influence of nanoparticle volume fraction.

- The Bejan number decreases for some larger values of $\mathrm{Br}$ and width of the channel and the distribution is relatively flat as the Brinkman number becomes smaller.

- Both entropy due to heat transfer and entropy due to fluid friction decrease with an increase in $\Omega$.

- The Bejan number increases and $\Phi$ decreases with an increase in $\Omega$.

\section{Acknowledgement}

The authors are grateful to the reviewers for their constructive remarks. One of the authors, B.J. Gireesha wishes to express his gratitude to University Grants Commission, New Delhi, India for the financial support under Raman Fellowship 2014-2015 for pursuing this work.

\section{Nomenclature}

$$
\begin{aligned}
\mathrm{Be} & - \text { Bejan number } \\
\mathrm{Br} & - \text { Brinkman number } \\
c_{p} & - \text { specific heat of fluid } \\
\mathrm{E}_{\mathrm{c}} & - \text { Eckert number } \\
E_{x} & - \text { electric field } \\
f_{v} & - \text { nanoparticle volume fraction } \\
h & - \text { height of channel from centerline to wall } \\
k & - \text { thermal conductivity } \\
k_{w} & - \text { Debye-Hükel parameter of the wall/liquid } \\
N_{F} & - \text { entropy due to fluid friction } \\
N_{H} & - \text { entropy due to heat transfer } \\
N_{s} & - \text { dimensionless entropy generation } \\
\mathrm{Pr} & - \text { Prandtl number } \\
r_{p} & - \text { particle radius size } \\
T_{0} & - \text { ambient temperature } \\
T_{l} & - \text { temperature of bottom plate } \\
T_{2} & - \text { temperature of top plate } \\
U & - \text { velocity of top plate } \\
u & - \text { fluid velocity } \\
u_{s} & - \text { steady state velocity } \\
u_{t} & - \text { transient velocity } \\
y & - \text { nondimensionalized } y \text { axis } \\
h & - \text { thermal diffusivity } \\
\alpha & - \text { permittivity of liquid } \\
\zeta_{w} & - \text { Zeta potential at the wall interface } \\
\lambda_{w} & - \text { Debye length } \\
&
\end{aligned}
$$




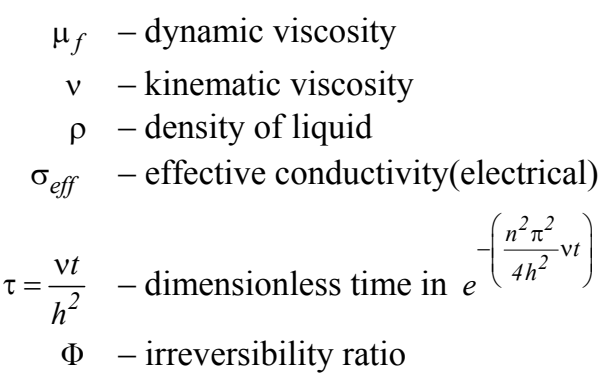

\section{References}

Abbassi H. (2007): Entropy generation analysis in a uniformly heated microchannel heat sink. - Energy, vol.32, pp.1932-1947.

Bejan A. (1996): Entropy Generation Minimization. - New York: CRC Press.

Chen K. (2005): Second law analysis and optimization of microchannel flows subject to different boundary conditions. - Int. J. Energy Res., vol.29, pp.249-263.

ChulJin Choi, Seok Pil Jang and Stephen U.S. Choi (2011): Electrokinetic effects of charged nanoparticles in microfluidic Couette flow. - Journal of Colloid and Interface Science, vol.363, pp.59-63.

Gorla R.S.R. (2014): Entropy generation in thermally fully developed electro-osmotic flow in circular microtubes. - Int. Jou. Microscale and Nanoscale Thermal and Fluid Transport Phenomena, vol.5, pp.279-296.

Groisman A. and Steinberg V. (1998): Mechanism of elastic instability in Couette flow of polymer solutions: Experiment. - Phys. Fluids, vol.10, pp.2451.

Guillermo Ibanez A and Sergio Cuevas (2010): Entropy generation minimization of a MHD (magnetohydrodynamic) flow in a microchannel. - Energy, vol.35, pp.4149-4155.

Kenney S., Poper K., Chapagain G. and Gordon F.C. (2013): Large Deborah number flows around confined microfluidic cylinders. - Rheol. Acta, vol.52, pp.485-497.

Omid Ejtehadi, Javad Abolfazli Esfahani and Ehsan Roohi (2012): Compressibility and rarefaction effects on entropy and entropy generation in micro/nano Couette flow using DSMC. - Journal of Physics: Conference Series, vol.362, pp.012008.

Osamah Haddad, Mohammad Abuzaid and Mohammad Al-Nimr (2004): Entropy generation due to laminar incompressible forced convection flow through parallel-plates microchannel. - Entropy, vol.6, No.5, pp.413-426.

Pathak J.A., Ross D. and Kalman B. Migler (2004): Elastic flow instability, curved streamlines, and mixing in microfluidic flows. - Phys. Fluids, vol.16, pp.4028.

Sheng Chen and Zhiwei Tian (2010): Entropy generation analysis of thermal micro-Couette flows in slip regime. International Journal of Thermal Sciences, vol.49, pp.2211-2221.

Soong C.Y. and Wang S.H. (2003): Theoretical analysis of electrokinetic flow and heat transfer in a microchannel under asymmetric boundary conditions. - Journal of Colloid and Interface Science, vol.265, pp.202-213.

Sunday C. Omowunmi and Xue-Feng Yuan (2013): Time-dependent non-linear dynamics of polymer solutions in microfluidic contraction flow - a numerical study on the role of elongational viscosity. - Rheol. Acta, vol.52, pp.337-354.

Wasif Ali Zahid, Youbing Yin and Ke-Qin Zhu (2007): Couette-Poiseuille flow of a gas in long microchannels. Microfluid Nanofluid, vol.3, pp.55-64. 